\title{
Enfisema subcutáneo masivo, nemotórax a tensión y neumomediastino tras traqueotomía percutánea
}

\author{
Massive subcutaneous emphysema, nemotórax voltage \\ and pneumomediastinum after percutaneous tracheostomy
}

\author{
Alberto Guillén $\mathbf{M}^{1}$, Rubén Moreno-Arrones $\mathrm{T}^{1}$, Clara Smilg N${ }^{1}$, Nathalie Fages $\mathbf{C}^{1}$, Dinis Antunes $\mathbf{D}^{1}$, \\ Iris Muñoz G', Marina Andreu G'1
}

\begin{abstract}
RESUMEN
Mujer de 68 años que ingresa en la Unidad de Cuidados Intensivos por shock séptico.

En el posoperatorio la paciente se mantiene inestable y se decide realizar traqueotomía percutánea (TP) por intubación prolongada. Al inicio la paciente presenta un enfisema subcutáneo que progresa hasta convertirse en masivo.

Se realiza TC torácico donde se observa pérdida de la morfología habitual de la pared posterior traqueal con solución de continuidad. Tras revisión mediante traqueobroncoscopía se decide colocar cánula de traqueotomía larga para dejar la lesión proximal al neumotaponamiento y así evitar la fuga de aire. Desde la colocación de la nueva cánula, la paciente presenta una disminución progresiva del enfisema hasta su total resolución.

La TP es un procedimiento seguro que se realiza con mucha frecuencia en los servicios de medicina intensiva, sin embargo, no está exenta de complicaciones.

En la revisión de Powell y cols describen las complicaciones de la TP destacando la inserción peritraqueal, la hemorragia, las infecciones de la herida, el neumotórax y la muerte.

El rango de complicaciones en la literatura oscila entre $3 \%$ y 18\%. Además, no se encuentran diferencias significativas respecto a las complicaciones entre la TP y la técnica abierta.
\end{abstract}

Palabras clave: Enfisema mediastínico, enfisema subcutáneo, neumotórax, traqueotomía.

\section{ABSTRACT}

A 68-year-old woman who enter in intensive care unit due to septic shock.

In the postoperative period, the patient remained unstable and decided to perform a percutaneous tracheotomy (PT) because prolonged intubation. In the first, the patient presents subcutaneous emphysema that progresses until becoming massive.

1 Médico de Hospital Santa Lucía, Cartagena.

Recibido el 29 de noviembre, 2016. Aceptado el 19 de marzo, 2017. 
Thoracic CT is performed where loss of the usual morphology of the posterior tracheal wall with continuity solution is observed. After revision by means of tracheobroncoscopia, it is decided to place a long tracheotomy cannula to leave the lesion proximal to pneumotaponamiento and thus avoid air leakage. From the placement of the new cannula, the patient presents a progressive decrease of the emphysema until its total resolution.

$P D$ is a safe procedure that is performed very frequently in the Intensive Care Services3, however, it is not without its complications.

The review of Powell et al 4 describes the complications of PT emphasizing peritracheal insertion, hemorrhage, wound infections, pneumothorax, and death.

The range of complications in the literature ranges from 3 to $18 \% 5$. In addition, no significant differences were found regarding the complications between the TP and the open technique.

Key words: Mediastinal emphysema, subcutaneus emphysema, pneumothorax, tracheotomy.

\section{INTRODUCCIÓN}

La traqueotomía percutánea (TP) fue desarrollada con el objetivo de reducir la morbilidad respecto a la técnica quirúrgica abierta. La primera TP fue descrita por Sheldon y Pudenz ${ }^{1}$ en 1957 y desde su inicio se han descrito numerosas variaciones de la técnica ${ }^{2}$.

La TP es un procedimiento generalmente seguro que se realiza con mucha frecuencia en los servicios de medicina intensiva ${ }^{3}$, sin embargo, no está exenta de complicaciones. En la revisión de Powell y cols ${ }^{4}$ se describen las complicaciones observadas con la TP, destacando la inserción peritraqueal, la hemorragia, las infecciones de la herida, el neumotórax y la muerte. El rango de complicaciones en la literatura oscila entre 3\% y $18 \%{ }^{5}$. Además, no se encuentran diferencias significativas respecto a las complicaciones entre la TP y la traqueotomía quirúrgica abierta.

El enfisema subcutáneo masivo es una complicación poco frecuente en cualquier traqueotomía y puede llegar a ser fatal. Está descrito en muy pocos casos en la literatura. El objetivo de este artículo es discutir un caso de enfisema subcutáneo masivo tras una traqueotomía percutánea.

\section{CASO CLÍNICO}

Mujer de 68 años con antecedentes de hipertensión, dislipemia e insuficiencia venosa crónica que

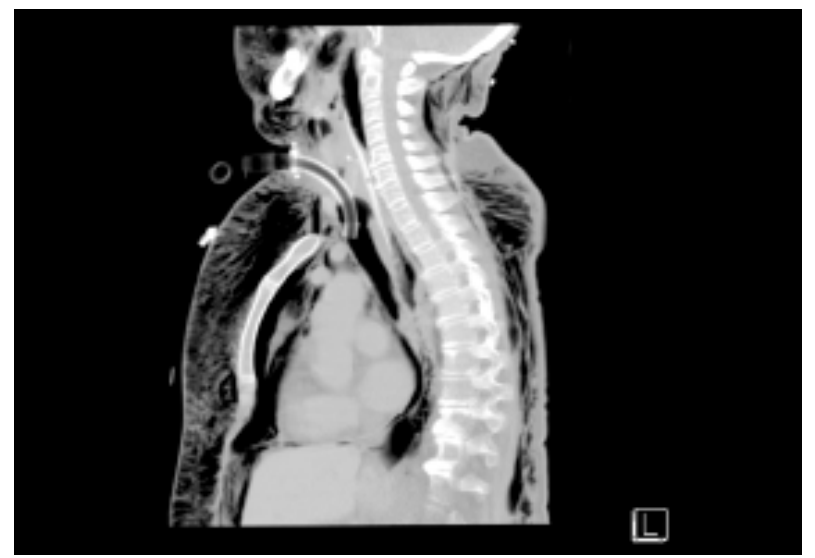

Figura 1. TC, corte sagital, se observa enfisema subcutáneo masivo, neumomediastino y solución de continuidad de la pared posterior traqueal. 


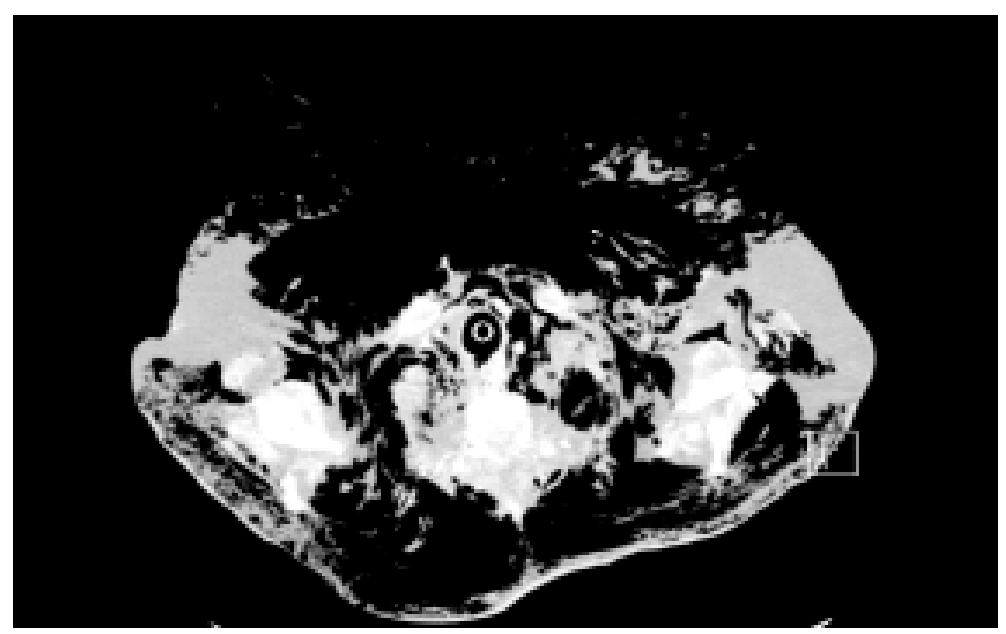

Figura 2. TC, corte axial, donde se identifica el enfisema que infiltra planos musculares profundos de la pared torácica junto con la afectación traqueal.

ingresa en la Unidad de Cuidados Intensivos (UCI) de nuestro hospital por shock séptico secundario a peritonitis fecaloídea por dehiscencia de sutura. Un mes antes había sido intervenida por perforación secundaria a plastrón apendicular.

En el posoperatorio en UCl, la paciente se mantiene inestable hemodinámicamente y requiere drogas vasoactivas a dosis altas. Se mantiene bajo ventilación mecánica invasiva y sedación.

Se decide realizar TP por intubación prolongada (Portex ${ }^{\circledR}$ GRIGGS $^{\circledR}$ Percutaneous Dilation Tracheostomy Kit). En las primeras horas tras el procedimiento, la paciente presenta un enfisema subcutáneo progresivo. Un día más tarde comienza de forma brusca con desaturación e hipotensión, todo ello compatible clínicamente con neumotórax a tensión. Se decide colocar tubo de tórax anterior izquierdo con mejoría posterior. Así mismo el enfisema subcutáneo progresa hasta convertirse en masivo.

Se realiza TC de tórax donde se observa una pérdida de la morfología habitual de la pared de la tráquea posterior con solución de continuidad. Tras la revisión por nuestra parte mediante traqueobroncoscopía se decide colocar cánula de traqueotomía extralarga (Bivona ${ }^{\circledR}$ Adult MidRange Aire-Cuf ${ }^{\circledR}$ ), para dejar la lesión proximal al neumotaponamiento y así evitar la fuga de aire. Desde la colocación de la nueva cánula, la paciente presenta una disminución progresiva del enfisema subcutáneo hasta su total resolución. Finalmente, se procede a la decanulación a los 35 días tras la TP.

\section{DISCUSIÓN Y CONCLUSIÓN}

El enfisema subcutáneo masivo tras la TP es una complicación rara que está descrita en la literatura en muy pocos casos.

Fish y cols ${ }^{6}$ describen un caso clínico donde la guía de dilatación Portex era la causante de la lesión encontrada en la pared traqueal posterior.

Douglas y Flabouris ${ }^{7}$ describen dos casos de enfisema subcutáneo, en ambos existía también una lesión en la pared posterior traqueal a nivel del tubo de traqueotomía, siendo la punta del introductor del tubo de traqueotomía la causante de las lesiones.

Kalye y cols ${ }^{8}$ refieren una laceración $1,5 \mathrm{~cm}$ en la tráquea membranosa en relación con el extremo distal a la cánula de traqueotomía. Determinan que la ventilación positiva a través del defecto de continuidad era la causa del enfisema subcutáneo del paciente. 
Fikkers y cols ${ }^{9}$ presentan la hipótesis de que el mal posicionamiento del tubo fenestrado de traqueostomía extraluminal podría ser uno de las mecanismos de enfisema subcutáneo. Igualmente, Lin y cols presentan dos casos de enfisema masivo tras técnica percutánea sin lesión traqueal y relacionados con la presión positiva a la que se sometieron los pacientes.

En nuestra paciente el enfisema subcutáneo se produjo varias horas después de la traqueotomía y tras haber llevado ventilación mecánica invasiva a través de la cánula.

La causa más frecuente de enfisema subcutáneo es la lesión de la pared traqueal. Nuestra paciente presentó un defecto en la pared traqueal posterior que fue origen del enfisema y de las complicaciones pulmonares posteriores. En nuestro caso, además, el enfisema pudo progresar de ma-

\section{BIBLIOGRAFÍA}

1. Sheldon C, Pudenz R. Percutaneous tracheotomy. JAMA 1957; 165: 2068-70.

2. TOYE F, WeINSTEIN J. A percutaneous tracheostomy device. Surgery 1969; 65: 384-9.

3. COOPER RM. Use and safety of percutaneous tracheostomy in intensive care. Report of a postal survey of ICU practice. Anaesthesia 1998; 53: 1209-27.

4. Powell DM, Price PD, Forrest LA. Review of percutaneous tracheostomy. Laryngoscope 1998; 108: 170-7.

5. Berrouchot, J, Oeku, J, Steinger, L, et al. Perioperative complications of percutaneous dilatational tracheostomy. Laryngoscope 1997; 107: 1539-44. nera más rápida al haberse sometido a la paciente a ventilación mecánica con presión positiva.

Cuando se produce una lesión traqueal es importante realizar una broncoscopía para comprobar otros daños en la vía respiratoria ${ }^{7}$, descartar lesiones esofágicas y determinar la distancia de la lesión respecto a la carina. En nuestra paciente la solución de continuidad estaba alejada de la carina y por ello se decidió cambiar la cánula por otra de mayor longitud para así sobrepasar el defecto endotraqueal. Además de no realizar este cambio, el movimiento del extremo distal de la cánula sobre la lesión podría empeorarla aún más.

La TP es un procedimiento en muchas ocasiones necesario, sobre todo en pacientes críticos, pero no así exenta de riesgo. Es importante conocer sus complicaciones ya que van asociadas a un aumento de la morbimortalidad.

6. Fish W, Bohiemer N, Cadle D, et al. A life threatening complication following percutaneous tracheostomy. Clin Intensive Care 1996; 7: 206-8.

7. Douglas W, Flabouris A. Surgical emphysema following percutaneous tracheostomy. Anaesth Intensive Care 1999; 27: 69-72.

8. Kaylie D, Wax M. Massive Subcutaneous Emphysema Following Percutaneous Tracheostomy. Am J Otolaryngol 2002; 23: 300-2.

9. Fikkers bG, van Veen JA, Kooloos JG, et AL. Emphysema and pneumothorax after percutaneous tracheostomy: case reports and an anatomic study. Chest 2004; 125: 1805-14.

10. Tin-Yu L, Chung-Jen H, Horng-Chyuan L. Massive subcutaneous enfisema following bronchoscopyguied percutaneous dilatational tracheostomy. $J$ Formos Med Assoc 2005; 104: 942-5.

Dirección: Rubén Moreno-Arrones T.

Hospital San Lucía, Cartagena

E mail: doc.rubentevar@gmail.com 